\title{
Connexin 43 SUMOylation improves gap junction functions between liver cancer stem cells and enhances their sensitivity to HSVtk/GCV
}

\author{
YIMENG SHEN $^{1 *}$, YANXIA LI ${ }^{2 *}$, XIAOFANG MA ${ }^{2}$, QIAOHAO WAN ${ }^{1}$, ZHONGMIN JIANG $^{3}$, \\ YIXIN LIU ${ }^{4}$, DIANYING ZHANG ${ }^{5}$, XIAOZHI LIU ${ }^{2}$ and WENHAN WU ${ }^{1}$ \\ ${ }^{1}$ Department of General Surgery, Peking University First Hospital, Beijing 100034; \\ ${ }^{2}$ Central Laboratory, and ${ }^{3}$ Department of Pathology, The Fifth Central Hospital of Tianjin, Tianjin 300450; \\ ${ }^{4}$ Department of Pathology, Tianjin Central Hospital of Gynecology and Obstetrics, Tianjin 300100; \\ ${ }^{5}$ Department of Orthopedics, Peking University People's Hospital, Beijing 100044, P.R. China
}

Received March 30, 2017; Accepted January 12, 2018

DOI: 10.3892/ijo.2018.4263

\begin{abstract}
Connexin $43(\mathrm{Cx} 43)$ can be modified and regulated by small ubiquitin-like modifier (SUMO)1; however, its role in liver cancer stem cells is poorly understood. In this study, we found a significant difference in the expression of $\mathrm{Cx} 43$ and SUMO1 between cancer stem cells and non-cancer stem cells in liver cancer. In liver cancer stem cells, CX43 was almost absent, although the level of SUMO1 was significantly higher than that in non-cancer stem cells. Further experiments confirmed that the conjugated site of $\mathrm{Cx} 43$ by SUMO1 was located in Lys-144 and Lys-237, both of which are highly conserved among species. By the co-expression of $\mathrm{Cx} 43$ and SUMO1 in cancer stem cells, the gap junction intercellular communication (GJIC) of liver cancer stem cells was obviously improved. Using this feature, we verified whether it could effectively increase the sensitivity of cancer stem cells to the herpes simplex virus 1 thymidine kinase (HSVtk) gene in combination with ganciclovir (GCV), a conventional chemotherapeutic drug, in vitro and in vivo. As expected, increasing the expression of Cx43 SUMOylation in liver cancer stem cells effectively enhanced their sensitivity to HSVtk/GCV. On the whole, this study revealed a novel method which may be used to effectively restore GJIC in cancer stem cells in liver cancer, which enhances their sensitivity to conventional chemotherapeutic drugs.
\end{abstract}

Correspondence to: Dr Wenhan Wu, Department of General Surgery, Peking University First Hospital, 8 Xishiku Street, Xicheng, Beijing 100034, P.R. China

E-mail: wuwenhan88@126.com

${ }^{*}$ Contributed equally

Key words: liver cancer, cancer stem cell, small ubiquitin-like modifiers, SUMOylation, connexin 43, herpes simplex virus 1 thymidine kinase/ganciclovir system

\section{Introduction}

Liver cancer is one of the most common malignant tumors of the digestive system (1). In China, the incidence of liver cancer has increased annually due to the persistent high infection rate of hepatitis B virus in the population (2). The major forms of liver cancer are hepatocellular carcinoma (HCC) and intrahepatic cholangiocarcinoma. In addition, there are two rare malignant liver tumors occurring in adolescence, hepatoblastoma and fibrolamellar carcinoma, both developing in a non-cirrhotic liver (3). At present, the treatment options for liver cancer and its prognosis depend on a number of factors, including tumor size, staging and the extent of liver injury (4). Surgical resection offers the optimal prognosis for long-term survival; however, only $10-15 \%$ of patients are considered suitable for surgical resection (5). The overall recurrence rate following resection is $50-60 \%$ (6). If the tumor cannot be removed completely, the disease is usually fatal within 3-6 months (5). Although radiotherapy and chemotherapy are often employed, the treatment effects are extremely limited (7).

An emerging theory in recent years is that the existence of cancer stem cells is the basic cause of the post-operative recurrence of liver tumors (8). The main obstacle is that these cancer stem cells are often in a state of dormancy which impairs the treatment effects of chemotherapeutic drugs (9). Some recent studies have shown that the herpes simplex virus thymidine kinase (HSVtk) gene and the complementary treatment with ganciclovir (GCV) exert marked antitumor effects against HCC by promoting apoptosis and inhibiting angiogenesis $(10,11)$. However, it is still difficult to prevent the recurrence of liver tumors (10-12).

It is well known that normal gap junction intercellular communication (GJIC) between tumor cells is crucial for HSVtk/GCV to exert its therapeutic effects (10). However, there are few studies on the structure and function of GJIC between liver cancer stem cells, although some studies have reported that their GJIC is obviously defective in liver tumors (13). Whether GJIC deficiency also occurs in liver cancer stem cells, and whether this feature promotes cancer 
stem cell resistance to chemotherapeutic drugs needs to be explored further.

Small ubiquitin-like modifier (SUMO)ylation is an essential post-translational modification that regulates a number of physiological and pathological events in cells, such as those involved in the stress response, nuclear translocation and the stabilization of protein structures (14). Although enhanced SUMOylation and the accumulation of SUMO-conjugated proteins have been widely observed in patients with various disorders, including cancers (15), their roles remain largely unknown. Recently, it has been reported that connexin 43 ( $\mathrm{Cx} 43)$, a protein involved in GJIC, can be covalently modified and regulated by SUMOylation in HeLa cells (16). However, to date, at least to the best of our knowledge, there are no studies available on the role of Cx43 SUMOylation in liver cancer stem cells, as well as no studies on whether Cx43 SUMOylation is triggered and plays roles in certain molecular events, such as stemness maintenance, immune escape and drug resistance, when cancer stem cells are exposed to different niches, or whether artificial Cx43 SUMOylation can be a clinical therapy for liver cancer stem cells.

Based on the above-mentioned issues, in this study, we detected the expression status of $\mathrm{CX} 43$ and SUMO1 in cancer stem cells of HCC and hepatoblastoma origin. To reveal the function of Cx43 SUMOylation in liver cancer stem cells, two exogenous plasmids, Cx43-wild-type (wt) and green fluorescent protein (GFP)-SUMO1, were co-transfected into hepatoblastoma-derived HepG2 cells, which are significantly less responsive to chemotherapy (17), and GJIC structure and function were examined. To further examine the effects of Cx43 SUMOylation on the effectiveness of chemotherapeutic drugs in liver cancer stem cells, we used HSVtk/GCV both in vitro and in vivo.

\section{Materials and methods}

Tissue specimens. Fresh surgical specimens (tumor and adjacent) were collected from 8 patients with liver cancer at the Department of General Surgery, the Fifth Central Hospital of Tianjin (Tianjin, China) between January, 2015 and December, 2015. The initial diagnosis of all frozen samples was completed by a senior pathologist, and their paraffinembedded sections were re-examined by another pathologist to confirm the initial diagnosis. This study was approved by the Ethics Committee of the Fifth Central Hospital of Tianjin. Written informed consent was obtained from all patients.

Cell culture. Two HCC cell lines (SNU182 and SNU449) and one hepatoblastoma-derived cell line (HepG2) were purchased from the American Type Culture Collection (Rockville, MD, USA) and cultured in Dulbecco's modified Eagle's medium supplemented with $10 \%$ fetal bovine serum (both from Invitrogen, Carlsbad, CA, USA), $100 \mathrm{U} / \mathrm{ml}$ penicillin and $100 \mu \mathrm{g} / \mathrm{ml}$ streptomycin (both from Sigma, St. Louis, MO, USA). Primary human normal liver cells were obtained from the left liver tissue of a 51-year-old male patient who underwent surgical resection for hepatic duct stones, which were cultured in Roswell Park Memorial Institute (RPMI)-1640 medium (Invitrogen) with $10 \%$ fetal bovine serum. All the cells were incubated at $37^{\circ} \mathrm{C}$ in a humidified $5 \% \mathrm{CO}_{2}$ atmosphere.
Isolation of liver cancer stem cells. CD133+ and CD133 HepG2 cells were obtained by fluorescence-activated cell sorting using fluorescence-activated cell sorting. These $\mathrm{CD}_{133^{+}}$and $\mathrm{CD} 133^{-}$cells were stained for $\mathrm{Cx} 43$ and SUMO1 proteins by immunofluorescence. The $\mathrm{CD} 133^{+}$cells were cultured in neurobasal medium containing $1 \mathrm{X}$ B27 (both from Invitrogen/Thermo Fisher Scientific, Inc., Carlsbad, CA, USA), $20 \mathrm{ng} / \mathrm{ml}$ basic fibroblast growth factor (Miltenyi Biotec, Bergisch Gladbach, Germany) and $20 \mathrm{ng} / \mathrm{ml}$ epidermal growth factor (Provitro Biosciences, Mt. Vernon, WA, USA), and incubated at $37^{\circ} \mathrm{C}$ with $5 \% \mathrm{CO}_{2}$. After $\sim 3$ weeks, cell spheres were collected, and immunofluorescence staining was performed to detect the expression of $\mathrm{CX} 43$ and SUMO1 proteins. The detailed method of immunofluorescence is described below.

Plasmids, transfection and treatment. The Ad-CMV-TK plasmid containing the HSVtk gene was provided by the Institute of Life Science, Nankai University (Tianjin, China). Lentiviral plasmids (pWPXLD-Cx43-wt, pWPXLD-GFP-SUMO1, pWPXLD-Cx43-K144R, pWPXLD-Cx43-K237R and pWPXLD-SENP1) were synthesized by Biogot Technology, Co. Ltd. (Nanjing, China). All constructs were verified by nucleic acid sequencing. The plasmids were then transfected into the $\mathrm{CD} 133^{+}$HepG 2 cells according to the experimental design, following the transfection protocols provided with the lentiviral plasmids. Finally, stably transfected cells were selected and cultured in stem cell culture medium until the formation of new clonal spheres. When the diameter of these spheres was at least $100 \mu \mathrm{m}, \mathrm{GCV}$ was added to the culture medium at $1 \mathrm{mg} / \mathrm{ml}$.

Immunoprecipitation. Total protein was extracted from the cells. For immunoprecipitation, $\sim 1 \mathrm{mg}$ of proteins was diluted 10-fold with Triton X-100 lysis buffer (50 mM Tris- $\mathrm{HCl}$, $\mathrm{pH} 7.5,150 \mathrm{mM} \mathrm{NaCl}, 1 \mathrm{mM}$ EDTA, $0.5 \%$ Triton $\mathrm{X}-100$, $1 \mathrm{mM}$ PMSF, $10 \mathrm{mM}$ iodoacetamide and protease inhibitors), pre-cleared with protein-agarose beads for $1 \mathrm{~h}$ at $4{ }^{\circ} \mathrm{C}$, followed by the addition of anti-Cx43 antibody (sc-59949, $2 \mu 1$ per $400 \mu \mathrm{g}$ of total protein; Santa Cruz Biotechnology, Dallas, TX, USA). Following incubation at $4^{\circ} \mathrm{C}$ overnight, immunoprecipitates were washed 3 times with $1 \mathrm{ml}$ Triton X-100 lysis buffer and then diluted in $2 \mathrm{X}$ SDS sample buffer. After boiling for $10 \mathrm{~min}$, the samples were evaluated by western blot analysis.

Western blot analysis. Total protein was extracted from fresh tissues or cells. Western blot analysis was performed using NuPAGE ${ }^{\mathrm{TM}}$ 4-12\% Bis-Tris Protein Gels (NP0336PK2; Invitrogen). Following gel electrophoresis, protein transfer and blocking, the membranes were incubated with antiSUMO1 (ab11672, 1:1,000; Abcam Trading Company Ltd., Shanghai, China) or anti-Cx43 (sc-59949, 1:2,000; Santa Cruz Biotechnology) antibodies overnight at $4^{\circ} \mathrm{C}$. The membranes were then incubated with the secondary antibodies [chicken anti-rabbit IgG conjugated with horseradish peroxidase (HRP) (sc-2955, 1:2,000) or chicken anti-goat IgG-HRP (sc-2953, 1:2,000) (both from Santa Cruz Biotechnology)] for $1 \mathrm{~h}$. A Super Signal protein detection kit (Pierce; Thermo Fisher Scientific, Inc.) was used to detect protein signals. The membranes were then stripped and re-probed with a goat anti-human $\beta$-actin polyclonal primary antibody $(1: 1,000$; 
Santa Cruz Biotechnology). All experiments were repeated 3 times with similar results.

Immunohistochemistry. The tumor tissues were fixed in $4 \%$ paraformaldehyde, washed with PBS, transferred to $70 \%$ ethanol and then embedded in paraffin in accordance with standard procedures (22). The antibodies used in this study were a goat anti-human Cx43 monoclonal antibody (ab87645, 1:2,000; Abcam Trading Company Ltd.) and mouse anti-human SUMO1 monoclonal antibody (sc-5308, 1:400; Santa Cruz Biotechnology).

Immunofluorescence staining. $\mathrm{CX} 43$ and SUMO1 protein expression was detected by immunofluorescence staining. Briefly, the cells were fixed with $4 \%$ paraformaldehyde (Sigma), treated with $3 \% \mathrm{H}_{2} \mathrm{O}_{2}$ for $10 \mathrm{~min}$, and then incubated overnight at $4^{\circ} \mathrm{C}$ with primary antibodies (anti-Cx43, sc-59949, 1:500; Santa Cruz Biotechnology; anti-SUMO1, ab11672, 1:400; Abcam Trading Company Ltd.) overnight at $4^{\circ} \mathrm{C}$, followed by incubation with Texas-Red or fluorescein isothiocyanate-labeled secondary antibodies (sc-2787, 1:500 dilution; Santa Cruz Biotechnology). Image-Pro Plus 6.0 software (Media Cybernetics, Rockville, MD, USA) was used for image analysis.

Lactate dehydrogenase $(\mathrm{LDH})$ activity detection. The $\mathrm{LDH}$ content in the conditioned medium was measured by an enzyme-linked immunosorbent assay using an LDH Activity assay kit (BioVision, Inc., Milpitas, CA, USA) in accordance with the manufacturer's instructions. Concisely, the culture medium was centrifuged at $400 \mathrm{x} \mathrm{g}$ at room temperature for $5 \mathrm{~min}$, and $20 \mu \mathrm{l}$ supernatant was then collected and mixed with $20 \mu \mathrm{l}$ 2,4-dinitrophenylhydrazine. Following incubation at $37^{\circ} \mathrm{C}$ for $15 \mathrm{~min}, 250 \mu \mathrm{l} \mathrm{NaOH}(0.4 \mathrm{M})$ was added to the mixture followed by incubation for a further $15 \mathrm{~min}$ at $37^{\circ} \mathrm{C}$. The mixture was maintained at room temperature for $5 \mathrm{~min}$, and the OD was subsequently recorded using a microplate reader at $450 \mathrm{~nm}$. The activity of $\mathrm{LDH}$ was derived from the OD values and expressed as U/1.

Apoptosis assay. Apoptosis was analyzed using an Annexin V/FITC apoptosis detection kit (BD Biosciences, Franklin Lakes, NJ, USA) according to the manufacturer's instructions. The cells were washed twice with cold PBS and then resuspended in $1 \mathrm{X}$ binding buffer at a concentration of $1 \times 10^{6}$ cells/ml. Subsequently, $100 \mu$ lof the solution $\left(1 \times 10^{5}\right.$ cells $)$ was transferred to a $5 \mathrm{ml}$ culture tube, followed by the addition of $5 \mu \mathrm{l}$ of FITC Annexin V and $5 \mu \mathrm{l}$ PI into the solution. The cells were gently vortexed and incubated for $15 \mathrm{~min}$ at room temperature $\left(25^{\circ} \mathrm{C}\right)$ in the dark. This was followed by the addition of $400 \mu \mathrm{l}$ of $1 \mathrm{X}$ binding buffer to each tube and analysis by flow cytometry within $1 \mathrm{~h}$.

Xenograft tumor assay. A total of 40 female nude mice (4 weeks old, weighing 14-16 g) were purchased from the Animal Center of the Academy of Military Medical Sciences (Beijing, China) and housed at the Experimental Animal Center of Tianjin Medical University under controlled temperature $\left(22-24^{\circ} \mathrm{C}\right)$ conditions with a $12 \mathrm{~h}$ light/dark cycle. All experimental procedures were carried out according to the regulations and internal biosafety and bioethics guidelines of the Tianjin Municipal Science and Technology Commission and were approved by the Animal Ethics Committee of the Fifth Central Hospital of Tianjin. The in vivo cancer model was established as previously described (18). The 40 nude mice were randomly divided into 5 groups as follows: i) The control group, in which HepG2 cell spheres obtained by the stable transfection of the HSVtk gene were transplanted into the subcutaneous tissues of the left shoulders of the nude mice, and the mice were then treated with PBS after 15 days; ii) the HSVtk group, in which HepG2 cell spheres obtained by the stable transfection of the HSVtk gene were transplanted, and after 15 days the mice were treated with $15 \mathrm{mg} / \mathrm{kg} \mathrm{GCV}$ every 2 days for 15 days; iii) the HSVtk + Cx43 group, in which HepG2 cell spheres obtained by stable transfection with both HSVtk and Cx43 genes were transplanted, and the mice were then treated with GCV in the same manner as described above after 15 days; iv) the HSVtk + SUMO1 group, in which HepG2 cell spheres obtained by the stable transfection with both HSVtk and SUMO1 genes were transplanted, and the mice were then treated with GCV in the same manner as described above after 15 days; v) the HSVtk + Cx $43+$ SUMO1 group, in which the HepG2 cell spheres obtained by the stable transfection of the HSVtk, SUMO1 and Cx43 genes were transplanted, and the mice were then treated with GCV in the same manner as described above after 15 days. Tumor growth was monitored by caliper measurements every 5 days for 30 days. Tumor volume (V) was calculated as follows: $\mathrm{V}=\mathrm{L} \times \mathrm{W}^{2} \times 0.5$ (L, length; W, width). Following observation, the mice were anesthetized by an intraperitoneal injection of chloral hydrate, according to the dose of $400 \mathrm{mg} / \mathrm{kg} / \mathrm{animal}$ body weight, and were then placed in sealed chambers where a flow rate of $25 \%$ volume/min of carbon dioxide gas are introduced for euthanasia. The weight of the mice upon sacrifice and of the tumors upon anatomy was detected separately. All tumor tissues blocks were prepared as paraffin-embedded sections for in situ apoptosis and immunohistochemical analyses. Terminal deoxynucleotidyl transferase (TdT)-mediated dUTP nick-end labeling (TUNEL) assay using the FLOWTAC kit (Trevigen, Gaithersburg, MD, USA) was used to examine cell apoptosis. The cells ( $10^{7}$ cells) were exposed to $2 \mathrm{mM}$ hydrogen peroxide $\left(\mathrm{H}_{2} \mathrm{O}_{2}\right)$ or $0.5 \mu \mathrm{g} / \mathrm{ml}$ triclosan for $4 \mathrm{~h}$ at $37^{\circ} \mathrm{C}$. The cells were then fixed in $1 \mathrm{ml} 3.7 \%$ formaldehyde for $10 \mathrm{~min}$ followed by permeabilization with Cytonin ${ }^{\mathrm{TM}}$ for $30 \mathrm{~min}$. The cells were then washed and resuspended in labeling reaction mix (TdT dNTP mix, TdT enzyme, $1 \mathrm{X} \mathrm{Mn}^{2+}$, $1 \mathrm{X}$ TdT labeling buffer) at $37^{\circ} \mathrm{C}$ for $1 \mathrm{~h}$. The reaction was terminated by the addition of $1 \mathrm{X}$ StopBuffer followed by staining with $25 \mathrm{ml}$ Strep-Fluorescein for $10 \mathrm{~min}$ in the dark. The cells were then stained with propidium iodide and RNase and analyzed using a FACSCanto cytometer (BD Biosciences).

Statistical analysis. All experiments were repeated at least 3 times. When the averages of the 2 groups was compared, the results were analyzed by the Student's t-test. The comparison of caverages among 3 or $>3$ groups were analyzed by one-way analysis of variance. Bonferroni correction was used to control the type I error. Data are expressed as the means \pm standard deviation (SD). All tests were two-tailed, and the level of statistical significance was set at $\mathrm{P} \leq 0.05$. GraphPad Prism 6 
A

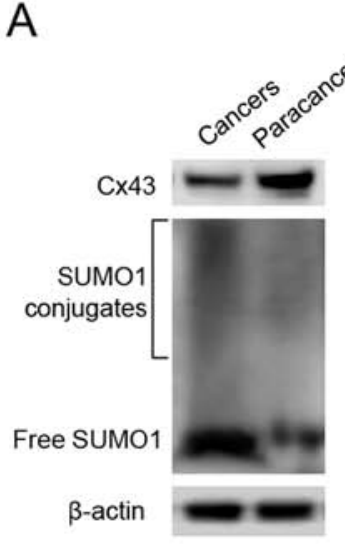

B

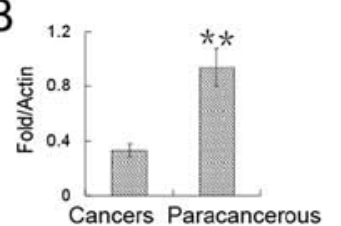

E

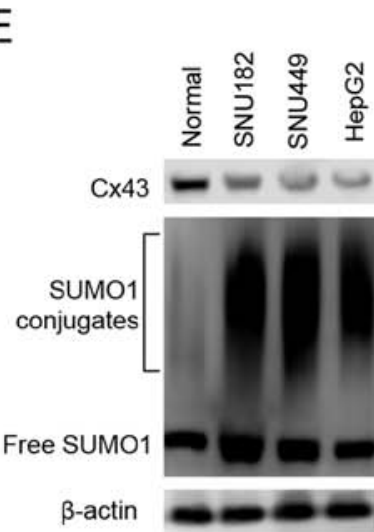

F

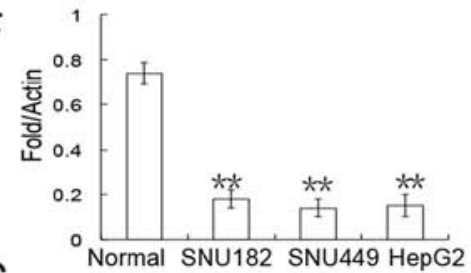

$G$

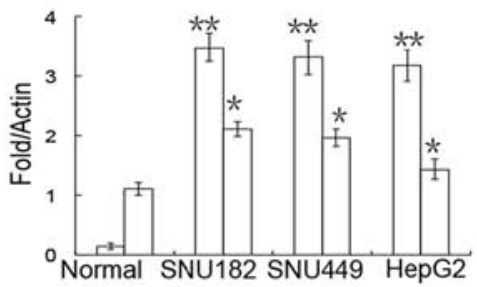

D
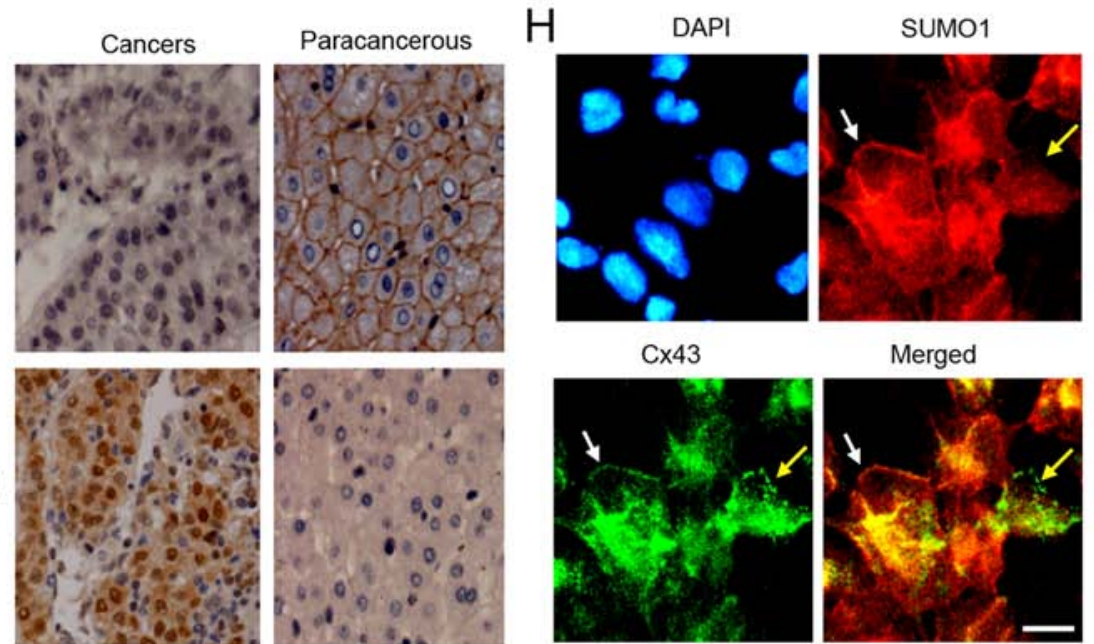

$\mathrm{H}$

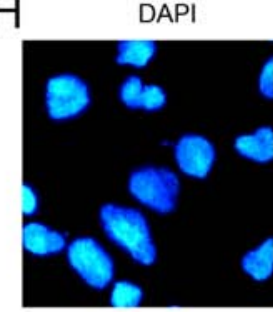

$\mathrm{C} \times 43$

asumO1 conjugates aFree SUMO1

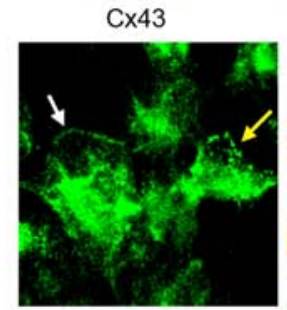

Merged

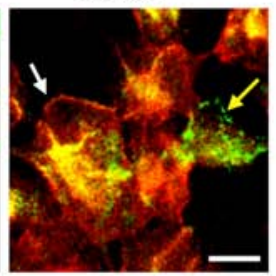

DAPI

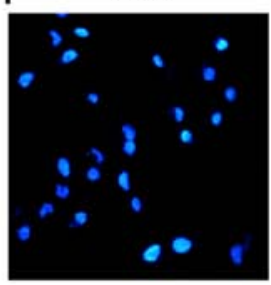

$\mathrm{Cx} 43$

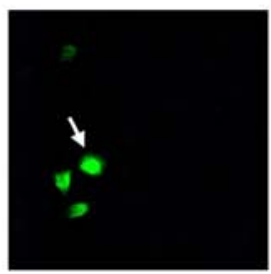

SUMO1

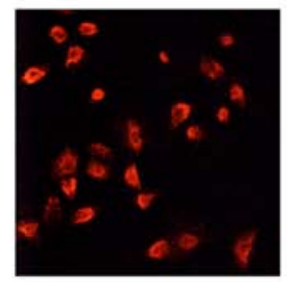

Merged

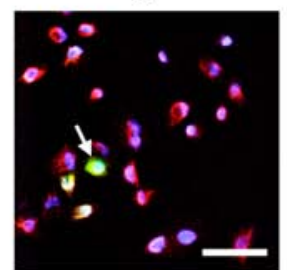

Figure 1. Expression of connexin 43 (Cx43) and small ubiquitin-like modifier (SUMO)1 proteins in liver cancer tissues and cell lines. (A) Expression of Cx43 and SUMO1 in liver tumors and adjacent tissues was detected by western blot analysis. (B) Quantitative analysis of the results shown in (A) for Cx43 expression. The level of $\mathrm{Cx} 43$ in liver tumor tissues was significantly lower than that in adjacent tissues. (C) Quantitative analysis of the results shown in (A) for SUMO1. The level of conjugated and free SUMO1 in liver cancer tissues was significantly higher than that in adjacent tissues. (D) The expression of Cx43 and SUMO1 was detected by immunohistochemistry. Cx43 protein was localized in the cell membrane of adjacent tissues, and SUMO1 was localized in the nucleus and cytoplasm of tumor cells (scale bar, $50 \mu \mathrm{m}$ ). (E) The expression of Cx43 and SUMO1 in liver cancer cell lines detected by western blot analysis. (F) Quantitative analysis of the results shown in (E) for Cx43. The expression level of Cx43 in hepatocellular carcinoma (HCC) and hepatoblastoma cell lines was significantly lower than that in normal liver cells. (G) Quantitative analysis of the results shown in (E) for SUMO1. The level of conjugated and free SUMO1 in the liver cancer cell lines was significantly higher than that in normal liver cells. (H) The expression of Cx43 (green) and SUMO1 (red) in normal liver cells detected by immunofluorescence. The white arrow indicates that Cx43 and SUMO1 were co-localized in the cell membrane. The yellow arrow indicates their separate locations (scale bar, $25 \mu \mathrm{m}$ ). (I) Expression of $\mathrm{Cx} 43$ (green) and SUMO1 (red) in HepG2 cells. The white arrow indicates that Cx43 and SUMO1 were co-localized in the same cell including the cytoplasm and membrane (scale bar, $100 \mu \mathrm{m}$ ). Graphs show the results of 3 independent experiments. Data represent the means $\pm \mathrm{SD} .{ }^{*} \mathrm{P}<0.05$ and ${ }^{* * *} \mathrm{P}<0.01$ compared with adjacent tissues or normal liver cells.

software (GraphPad Software, Inc., La Jolla, CA, USA) was used for all statistical tests.

\section{Results}

The expression of CX43 and SUMO1 differs markedly between liver cancer cells and normal liver cells. Previous studies have reported a relatively high frequency of $\mathrm{Cx} 43$ expression loss in many types of tumors, such as glioma and breast, colorectal, prostate, lung and pancreatic cancers (19-21). Therefore, in this study, we first examined the expression of Cx43 in liver cancer tissues and adjacent tissues from 8 patients who underwent surgical resection. The results of western blot analysis revealed that the expression of $\mathrm{Cx} 43$ in the liver cancer tissue was significantly lower than that in the adjacent (paracancerous) tissue by $\sim 0.3$-fold (Fig. 1A and B). Further immunohistochemical analyses revealed that $\mathrm{Cx} 43$ was mainly expressed in the liver cell membranes of the normal tissues adjacent to the cancer tissues (Fig. 1D). Furthermore, in the cancer nest, Cx43 expression was obviously weak (Fig. 1D).

Similar trends were detected in the liver cancer cell lines. Compared with the normal liver cells, there was a marked downregulation of $\mathrm{Cx} 43$ expression in these 3 liver cancer cell lines (Fig. 1E and F). Immunofluorescence staining revealed that $\mathrm{Cx} 43$ was localized in the cell membranes of the normal hepatocytes (Fig. 1H). Compared with the normal liver cells, only a small number $(<30 \%)$ of HepG2 liver cancer cells expressed Cx43, and the GJIC structure in the cell membrane was almost unseen (Fig. 1I).

To verify the correlation between SUMO1 and Cx43 expression, SUMO1 expression was detected in the liver tumors and liver cancer cell lines. Our results revealed that the expression level of SUMO1 in both free and conjugated states was significantly higher in the liver cancer tissues and liver 
A

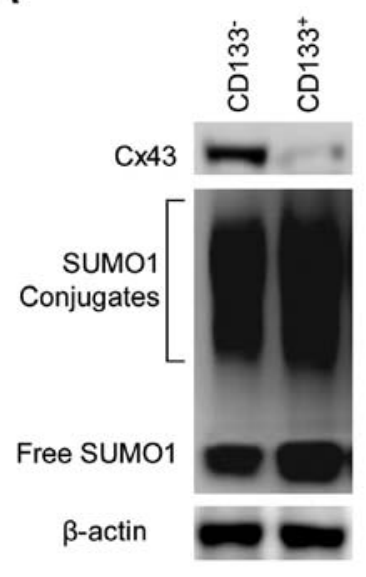

D

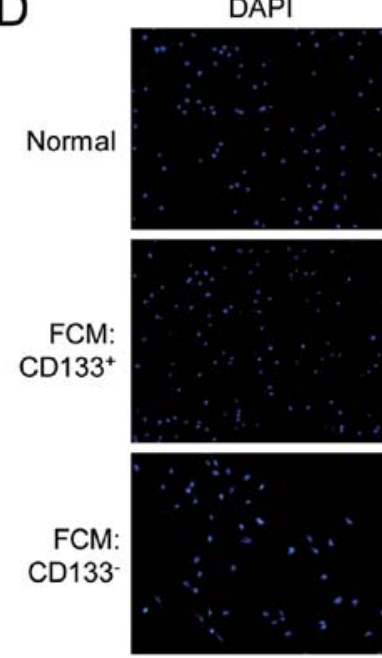

SUMO1
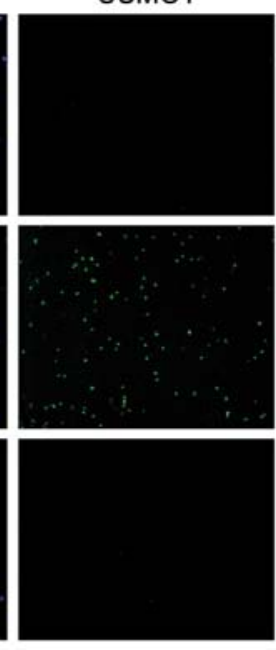

E
DAPI

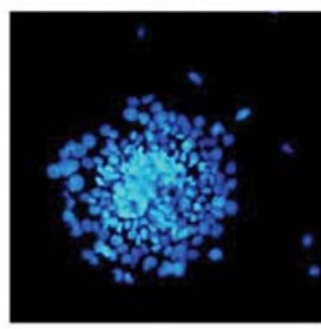

$\mathrm{Cx} 43$

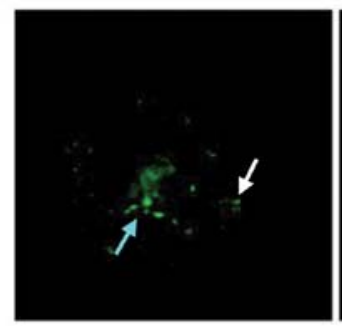

Cx43

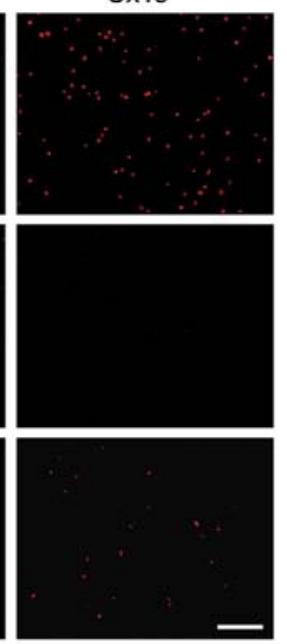

SUMO1

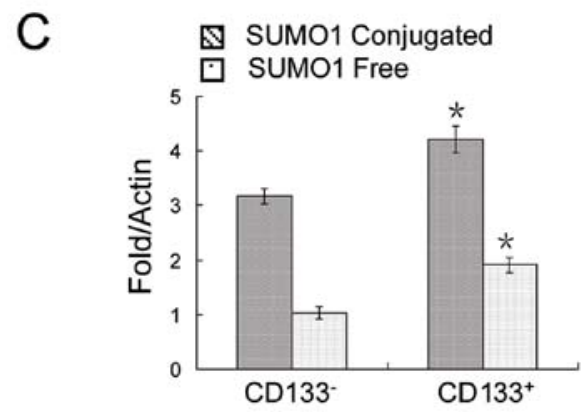

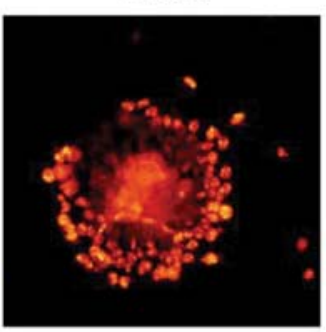

Merged

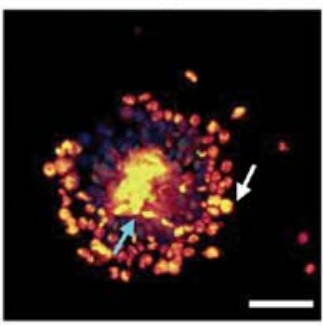

Figure 2. The expression of connexin 43 (Cx43) and small ubiquitin-like modifier (SUMO)1 proteins in cancer stem cells and non-cancer stem cells in liver cancer. (A) The expression of Cx43 and SUMO1 was detected by western blot analysis. (B) Quantitative analysis of the results shown in (A) for Cx43. Cx43 was almost absent in the liver cancer stem cells. (C) Quantitative analysis of the results shown in (A) for SUMO1. The level of conjugated and free SUMO1 in liver cancer stem cells was significantly higher than that in non-cancer stem cells. (D) Immunofluorescence staining of cells sorted by florescence-activated cell sorting (scale bar, $200 \mu \mathrm{m}$ ). (E) Expression of Cx43 and SUMO1 in HepG2 cell spheres. Arrows indicate the co-localization of the two proteins (scale bar, $100 \mu \mathrm{m})$. Graphs show data of 3 independent experiments. Data represent the means $\pm \mathrm{SD}$. ${ }^{*} \mathrm{P}<0.05$ and ${ }^{* *} \mathrm{P}<0.01$ compared with CD133 cells.

cancer cell lines than in the adjacent tissues and normal liver cells (Fig. 1A, C, E and G). Of note, we found no obvious correlation between the expression of SUMO1 and CX43 in both normal liver cells and liver cancer cells, as CX43 and SUMO1 expression was co-localized in the cell membrane (Fig. 1H, white arrow) but was also expressed separately (Fig. 1H, yellow arrow).

CX43 and SUMO1 expression patterns are distinctly different between cancer stem cells and non-cancer stem cells. To analyze the expression patterns of $\mathrm{Cx} 43$ and SUMO1 in cancer stem cells and non-cancer stem cells, CD133 ${ }^{+}$and CD133HepG2 cells were assessed by flow cytometry. The results revealed a very weak Cx43 expression, but a strong SUMO1 expression in the CD133+ HepG2 cells (Fig. 2A-C). The same pattern was observed by immunofluorescence staining of the cells sorted by fluorescence-activated cell sorting (Fig. 2D).
Finally, we examined their expression in HepG2 cell spheres. The results revealed that $\mathrm{Cx} 43$ was expressed in only a very small number of cells in clonal spheres (Fig. 2E).

CX43 is modified by SUMO1 in liver cancer stem cells. Although Kjenseth et al (16) reported that $\mathrm{Cx} 43$ was covalently modified and regulated by SUMOylation in HeLa cells, it is not known whether the same mechanism exists in cancer stem cells. Therefore, in this study, CD133 ${ }^{+}$HepG2 cells were transfected with $\mathrm{Cx} 43$-wt alone or in combination with GFP-SUMO1. Cell lysates were subjected to immunoprecipitation using an anti-Cx43 antibody or preimmune serum as a negative control. SUMOylated Cx43 was detected by western blot analysis using an anti-GFP antibody. The results revealed that only Cx43-wt and GFP-SUMO1 co-transfection led to the appearance of a clear band near $55 \mathrm{kDa}$ indicating SUMOylated Cx43 (Fig. 3A). 

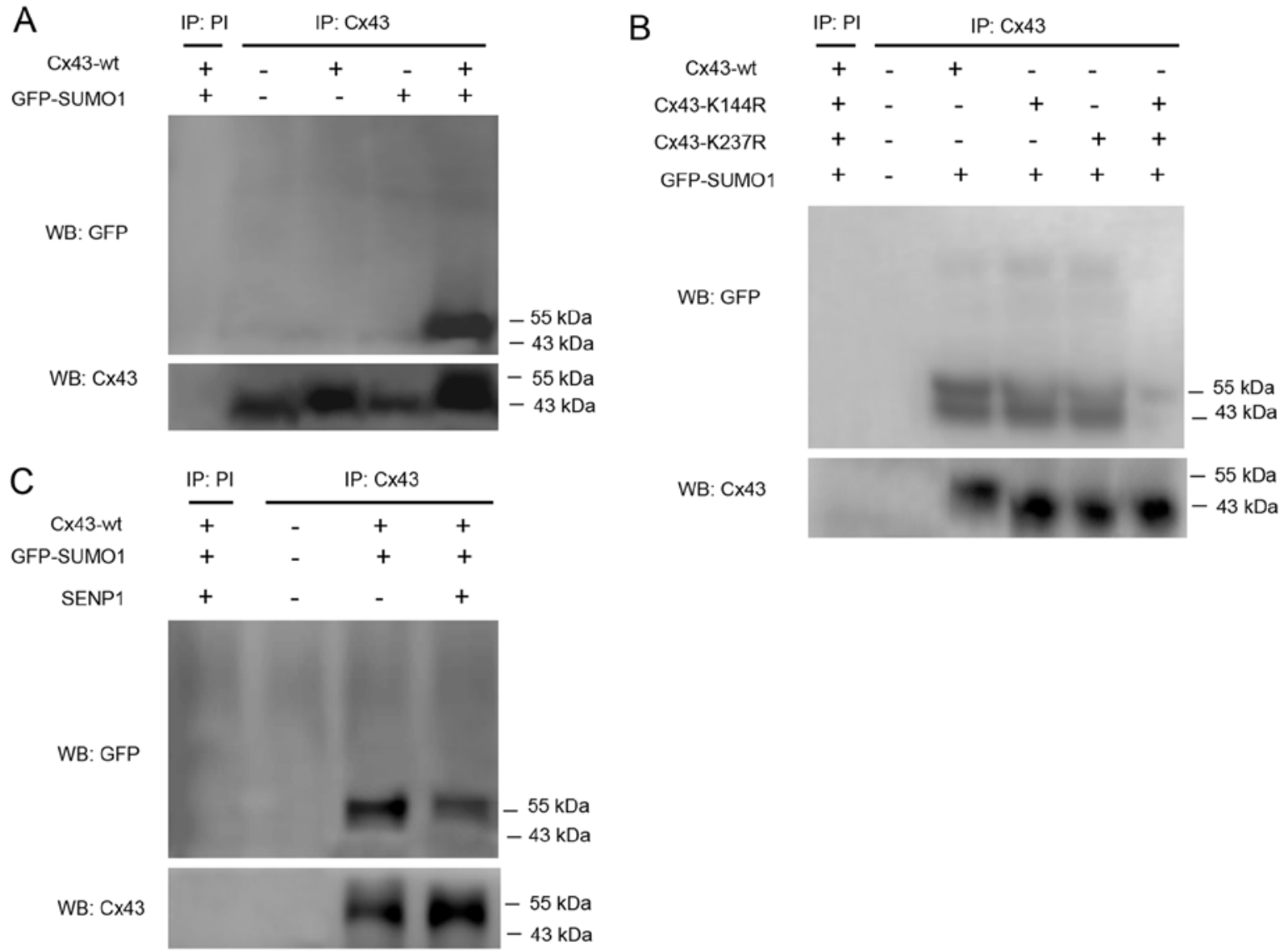

Figure 3. Connexin 43 (Cx43) is modified by small ubiquitin-like modifier (SUMO)1 in liver cancer stem cells. (A) CD133 ${ }^{+}$HepG2 cells were transfected with Cx43-wt alone or in combination with green fluorescent protein (GFP)-SUMO1. Cell lysates were subjected to immunoprecipitation (IP) using an anti-Cx43 antibody or preimmune serum (PI) as a negative control. SUMOylated Cx43 was detected by western blot analysis (WB) using an anti-GFP antibody. The blot was stripped and re-probed using an anti-Cx43 antibody. (B) CD133 ${ }^{+} \mathrm{HepG} 2$ cells were transfected with Cx43-wt, Cx43-K144R, Cx43-K237R, or Cx43-K144R and Cx43-K237R in combination with GFP-SUMO1 as indicated. (C) CD133 ${ }^{+}$HepG2 cells were transfected with Cx43-wt, GFP-SUMO1 in combination with SENP1 as indicated.

Kjenseth et al (16) also reported that the covalent binding site of Cx43 modified by SUMO1 was located at Lys-144 and Lys-237. Based on the high conservation of these two loci among species, we hypothesized that the covalent binding of Cx43 to SUMO1 may occur at the same site in liver cancer stem cells. Therefore, two mutants, Cx43-K144R and Cx43-K237R, were co-transfected with GFP-SUMO1. The results confirmed that the two mutations were indeed able to abolish the covalent binding of SUMO1 to Cx43 (Fig. 3B).

SENP1 induces the de-conjugation of SUMOs, particularly SUMO1, from the substrate protein, which was detected to reveal whether it is capable of dissociation of conjugated SUMO1 from Cx43. The results confirmed that SENP1 partially, but not completely, counteracted the conjugation of SUMO1 to Cx43 (Fig. 3C). However, the in-depth reasons require further investigation.

CX43 SUMOylation increases GJIC among liver cancer stem cells. To further investigate whether Cx43 SUMOylation increases GJIC in liver cancer stem cells, we observed the transmission of Hoechst 33342 in HepG2 cell spheres that were co-transfected with $\mathrm{Cx} 43$-wt alone or in combination with GFP-SUMO1. In theory, cancer stem cells are able to efflux the fluorescent dye, Hoechst 33342 (22). Therefore, fluorescence microscopic observations or flow cytometry should detect no staining. Our results revealed that when the cancer stem cells underwent $\mathrm{CX} 43$ and SUMO1 gene transfection, they were able to efflux most of the dye. However, when they were transfected with the Cx43 or SUMO1 genes alone, the amount of dye in the clonal spheres increased significantly. We hypothesized that SUMO1 transfection alone may induce some unknown events, so that the degree of dye diffusion among the cells was increased. The specific mechanisms require further investigation. We found that when the cells were co-transfected with SUMO1 and Cx43, the spread of the dye in clonal spheres was increased significantly, which was the highest in all test groups (Fig. 4A).

CX43 SUMOylation increases the sensitivity of liver cancer stem cells to HSVtk/GCV. We then applied this mechanism of increased GJIC by $\mathrm{Cx} 43$ SUMOylation to enhance the sensitivity of cancer stem cells to a conventional drug system, HSVtk/GCV, for which there no reports available to date to prove its effectiveness for cancer stem cells; however, it has been shown to be effective for most cancer cells (12). The results from the LDH content and flow cytometric assays both confirmed the obvious damage and apoptosis of the tumor cells by co-transfection of the Cx43 and SUMO1 genes (Fig. 4B and C). Subsequently, the cell spheres were prepared as frozen sections and subjected immunofluorescence staining or in situ apoptosis detection. 


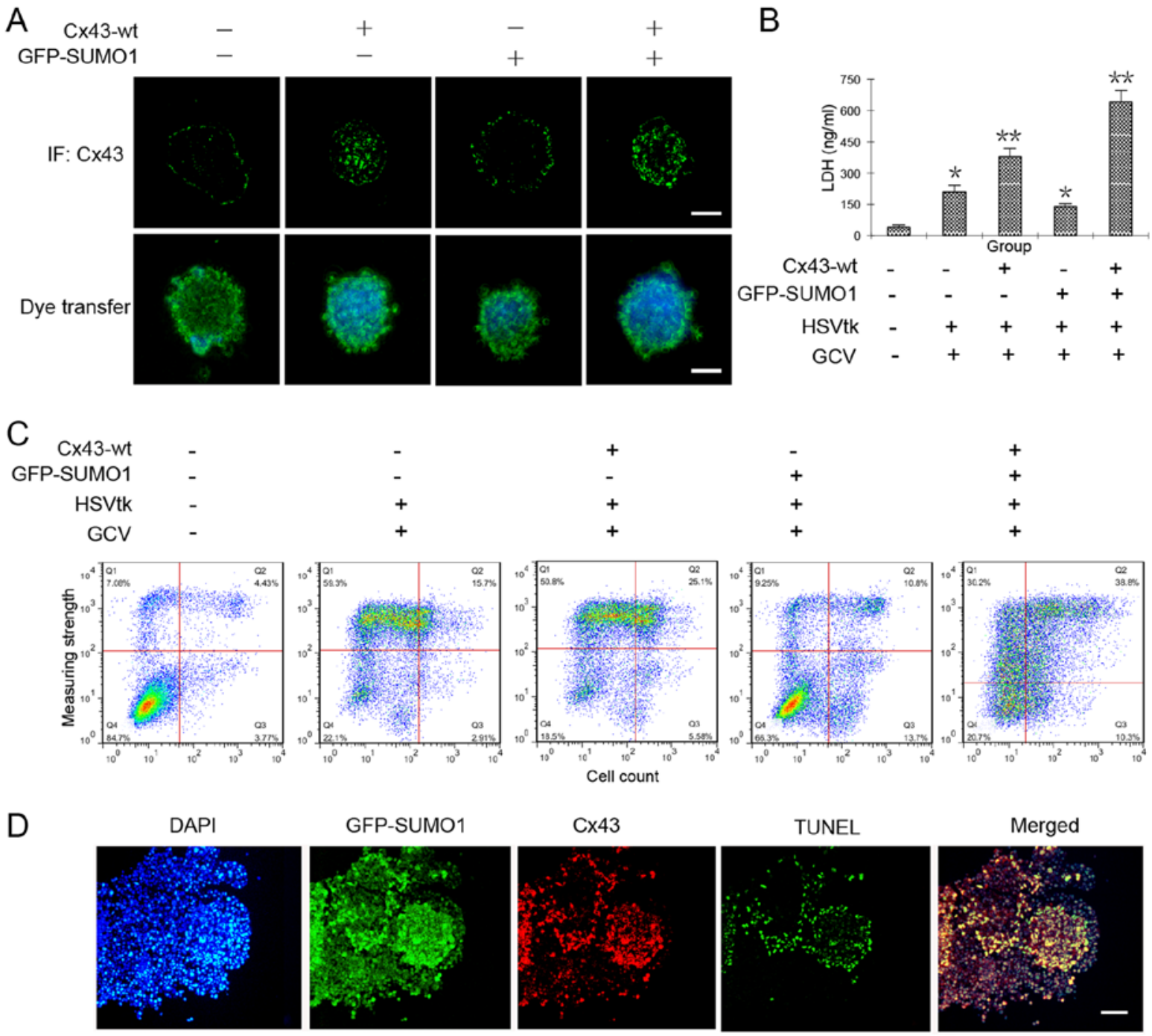

Figure 4. Connexin 43 (Cx43) small ubiquitin-like modifier (SUMO)ylation may improve the gap junction intercellular communication (GJIC) of liver cancer stem cells and enhance their sensitivity to HSVtk/ganciclovir (GCV) in vitro. (A) CD133 ${ }^{+} \mathrm{HepG} 2$ cells were transfected with Cx43-wt alone or in combination with green fluorescent protein (GFP)-SUMO1, and then cultured as clonal spheres in stem cell culture medium. Cx43 expression was observed by detecting the green fluorescence of GFP. Transfer of the dye was observed by detecting blue fluorescence of Hoechst 33342 (scale bar, $100 \mu \mathrm{m}$ ). (B) LDH content was measured by an enzyme-linked immunosorbent assay after clonal spheres were subjected HSVtk/GCV treatments. (C) Apoptosis was detected by flow cytometry. (D) Clonal spheres were frozen, and then immunofluorescence was performed to detect cell apoptosis in situ (scale bar, $50 \mu \mathrm{m}$ ). Graphs show the data of 3 independent experiments. Data represent the means \pm SD. ${ }^{*} \mathrm{P}<0.05$ and ${ }^{* *} \mathrm{P}<0.01$ compared with the controls.

The results revealed that cellular damage mainly occurred in the Cx43 and SUMO1 co-expression sites (Fig. 4D).

\section{CX43 SUMOylation enhances the sensitivity of subcutaneous} tumors in immunodeficient mice to $H S V t k / G C V$. To verify whether the enhanced sensitivity to HSVtk/GCV therapy in liver cancer stem cells also occurs in vivo, immunodeficient mice were employed in this study. Considering the changes in the growth state of liver cancer stem cells induced by $\mathrm{Cx} 43$ or SUMO1 alone in vitro, measurements of subcutaneous tumor volumes were performed at 2 time periods. During the first 15 days, the animals were not administered GCV, and the tumor volume was recorded. The results revealed that transfection of the HSVtk or SUMO1 genes alone did not induce significant differences in tumor volumes compared with the control group. However, transfection with $\mathrm{Cx} 43$ alone delayed tumor growth (Fig. 5A). Next, GCV was administered on day 15 following tumor sphere injection. The results revealed that the tumor stem cells transfected with the different genes exhibited different therapeutic responses to GCV. The degree of their sensitivity to GCV treatment was as follows: The control group $<$ SUMO1 group $<$ HSVtk group $<$ Cx43 group $<$ Cx43 + SUMO1 group (Fig. 5B). The results were consistent with the results of tumor weight measurements (Fig. 5C) and the rate of apoptosis in tumor tissues (Fig. 5D).

\section{Discussion}

The expression of $\mathrm{Cx} 43$ is reduced or even absent in a variety of tumors, including liver cancer $(13,23,24)$. On the other hand, various tumors highly express SUMO1, and this trend is increased with the degree of malignancy (24-26). However, to date, at least to the best of our knowledge, limited research has been performed into the association between $\mathrm{Cx}_{4} 43$ and SUMO1 in liver cancer. In this study, we first examined the expression of $\mathrm{Cx} 43$ and SUMO1 in liver tumor and adjacent 

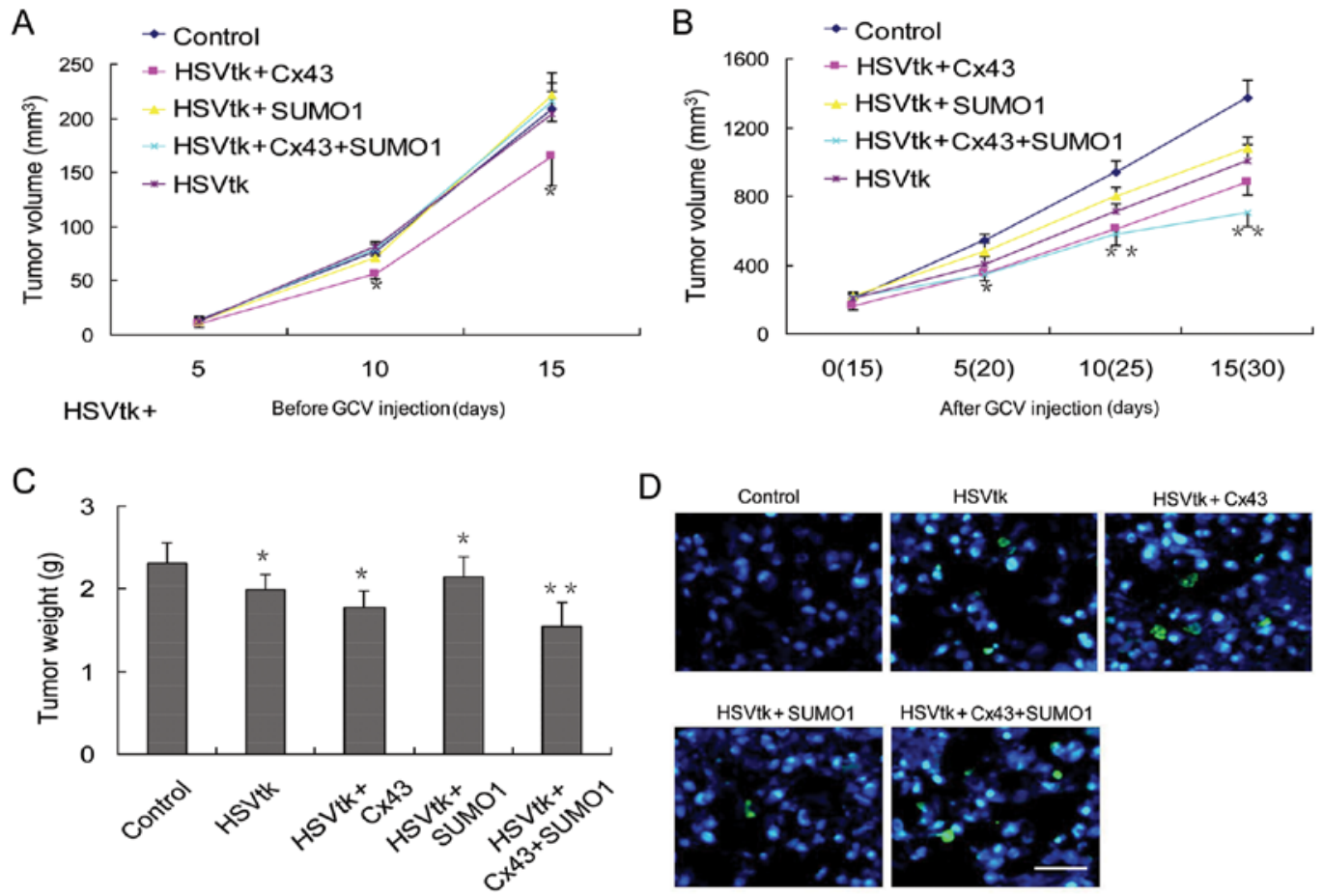

Figure 5. Connexin 43 (Cx43) small ubiquitin-like modifier (SUMO)ylation enhances the sensitivity of liver cancer stem cells to HSVtk/ ganciclovir (GCV) in vivo. (A) Tumor volumes were monitored for 15 days prior to GCV injection. (B) Tumor volumes were monitored for 15 days after GCV injection. (C) Tumor weight was determined at the end of the experiments. (D) Apoptosis in xenografted tumor sections was detected by terminal deoxynucleotidyl transferase (TdT)-mediated dUTP nick-end labeling (TUNEL) assay (scale bar, $100 \mu \mathrm{m}$ ). Graphs show the data of 3 independent experiments. Data represent the means $\pm \mathrm{SD} .{ }^{*} \mathrm{P}<0.05$ and ${ }^{* *} \mathrm{P}<0.01$ compared with the controls.

tissues. The results revealed a negative correlation between CX43 and SUMO1 expression. Further results from 3 liver cancer cell lines confirmed this conclusion. However, the results from immunocytochemistry revealed that $\mathrm{Cx} 43$ and SUMO1 proteins were not always co-located in the cell membrane, suggesting that the binding of the two proteins may be temporary, and their interaction is produced only when the function is performed jointly.

We then examined the protein expression of $\mathrm{Cx} 43$ and SUMO1 in liver cancer stem cells. The results revealed that Cx43 protein was almost completely absent in the liver cancer stem cells, which implies that intercellular communication is obviously absent between liver cancer stem cells. However, the expression of SUMO1 in liver cancer stem cells was obviously much higher than that in non-cancer stem cells. It has been reported that several proteins, such as octamerbinding transcription factor-4 (Oct-4), hypoxia-inducible factor-1 $\alpha(\mathrm{HIF}-1 \alpha)$ and AKT1, which play key roles in maintaining the stemness of cancer stem cells, are regulated by SUMO1 (27-30). This may partly explain the reasons for the high expression level of SUMO1 in liver cancer stem cells.

Kjenseth et al reported that $\mathrm{Cx} 43$ is a target protein modified by SUMO1 in HeLa cells (16). Therefore, in this study, we confirmed the above finding in liver cancer stem cells, and that SUMO binding sites in the Cx43 protein were Lys-144 and Lys-237. This result implies that Cx43 SUMOylation is highly conserved among species and may have a wide range of applications in several areas in the future.

The main purpose of this study was to observe the effect of Cx43 SUMOylation on the recovery of GJIC in liver cancer stem cells and use this feature to restore the sensitivity of cancer stem cells to chemotherapeutic drugs. Therefore, HepG2 cells were selected as the research object due to its more pronounced resistance to chemotherapeutic drugs than other two HCC cell lines. The results revealed that $\mathrm{Cx} 43$ SUMOylation effectively improved GJIC in liver cancer stem cells and increased their sensitivity to the chemotherapeutic drug system, HSVtk/GCV. This finding provides us with a new strategy for the treatment of liver tumors.

In addition to the above-mentioned findings, we observed that $\mathrm{Cx} 43$ gene transfection alone partially inhibited tumor growth in mice. This result is consistent with previous reports that considered $\mathrm{Cx} 43$ as a tumor suppressor gene (31-33). However, it is clear that the inhibitory effect of $\mathrm{Cx} 43$ alone on tumor growth is extremely limited $(31,33)$. Similarly, the killing effect of HSVtk/GCV alone on liver cancer cells is limited, particularly in $\mathrm{CD}_{133^{+}}$cell spheres that were cultured in vitro. However, the induction of Cx43 SUMOylation significantly enhanced the sensitivity of the liver cancer stem cells to HSVtk/GCV.

In conclusion, we found a novel method which may be used to restore GJIC in liver cancer stem cells, which significantly enhances their sensitivity to chemotherapeutic drugs. Based on the high conservation of Cx43 SUMOylation among species, it may be extended to more therapeutic areas in the future.

\section{Acknowledgements}

This study was supported by the National Natural Science Foundation of China (grant nos. 81471175 and 1671246), and the Tianjin Health Bureau Science and Technology Projects (grant nos. 2014KY23 and 2015KZ018). 


\section{Competing interests}

The authors declare that they have no competing interests.

\section{References}

1. Kudo M, Trevisani F, Abou-Alfa GK and Rimassa L: Hepatocellular carcinoma: Therapeutic guidelines and medical treatment. Liver Cancer 6: 16-26, 2016.

2. Wang Z, Li Z, Ye Y, Xie L and Li W: Oxidative stress and liver cancer: Etiology and therapeutic targets. Oxid Med Cell Longev 2016: 7891574, 2016.

3. Castelli G, Pelosi E and Testa U: Liver cancer: Molecular characterization, clonal evolution and cancer stem cells. Cancers (Basel) 9: E127, 2017.

4. Salem AI and Winslow ER: Current technical aspects of oncological hepatic surgery. Hepatobiliary Pancreat Dis Int 16: $147-154,2017$

5. Kim PT, Jang J, Fischer S, Greig PD, Gallinger S, Wei AC, McGilivray IM, Cattral MS and Cleary SP: Liver resection for multifocal hepatocellular carcinoma. J Clin Oncol 30 (Suppl 4): $355,2012$.

6. Cong WM and Wu MC: New insights into molecular diagnostic pathology of primary liver cancer: Advances and challenges. Cancer Lett 368: 14-19, 2015.

7. Büttner N, Schmidt N and Thimme R: Perspectives of immunotherapy in hepatocellular carcinoma (HCC). Z Gastroenterol 54: 1334-1342, 2016.

8. Nishida N, Kitano M, Sakurai T and Kudo M: Molecular mechanism and prediction of sorafenib chemoresistance in human hepatocellular carcinoma. Dig Dis 33: 771-779, 2015

9. Watson ME, Diepeveen LA, Stubbs KA and Yeoh GC: Glycosylation-related diagnostic and therapeutic drug target markers in hepatocellular carcinoma. J Gastrointestin Liver Dis 24: 349-357, 2015

10. Wu K, Yang L, Huang Z, Zhao H, Wang $\mathrm{J}$ and $\mathrm{Xu} \mathrm{S}$ : A double suicide gene system driven by vascular endothelial growth factor promoter selectively kills human hepatocellular carcinoma cells. Oncol Lett 11: 3152-3160, 2016.

11. Liu X, Wang S, Guo X, Wei F, Yin J, Zang Y, Li N and Chen D: Exogenous p53 and ASPP2 expression enhances rAdV-TK/GCV-induced death in hepatocellular carcinoma cells lacking functional p53. Oncotarget 7: 18896-18905, 2016.

12. Park JH, Kim KI, Lee KC, Lee YJ, Lee TS, Chung WS, Lim SM and Kang JH: Assessment of $\alpha$-fetoprotein targeted HSV1-tk expression in hepatocellular carcinoma with in vivo imaging. Cancer Biother Radiopharm 30: 8-15, 2015.

13. Yang Y, Qin SK, Wu Q, Wang ZS, Zheng RS, Tong XH, Liu H, Tao L and He XD: Connexin-dependent gap junction enhancement is involved in the synergistic effect of sorafenib and all-trans retinoic acid on HCC growth inhibition. Oncol Rep 31: 540-550, 2014

14. Da Silva-Ferrada E, Ribeiro-Rodrigues TM, Rodríguez MS and Girão H: Proteostasis and SUMO in the heart. Int J Biochem Cell Biol 79: 443-450, 2016.

15. Bettermann K, Benesch M, Weis S and Haybaeck J: SUMOylation in carcinogenesis. Cancer Lett 316: 113-125, 2012.

16. Kjenseth A, Fykerud TA, Sirnes S, Bruun J, Yohannes Z, Kolberg M, Omori Y, Rivedal E and Leithe E: The gap junction channel protein connexin 43 is covalently modified and regulated by SUMOylation. J Biol Chem 287: 15851-15861, 2012.
17. López-Terrada D, Cheung SW, Finegold MJ and Knowles BB: Hep G2 is a hepatoblastoma-derived cell line. Hum Pathol 40: 1512-1515, 2009.

18. Liu Z, Jiang Z, Huang J, Huang S, Li Y, Yu S, Yu S and Liu X: miR-7 inhibits glioblastoma growth by simultaneously interfering with the PI3K/ATK and Raf/MEK/ERK pathways. Int J Oncol 44: 1571-1580, 2014.

19. Sin WC, Crespin S and Mesnil M: Opposing roles of connexin43 in glioma progression. Biochim Biophys Acta 1818: 2058-2067, 2012.

20. Grek CL, Rhett JM, Bruce JS, Ghatnekar GS and Yeh ES: Connexin 43, breast cancer tumor suppressor: Missed connections? Cancer Lett 374: 117-126, 2016.

21. Leithe E: Regulation of connexins by the ubiquitin system: Implications for intercellular communication and cancer. Biochim Biophys Acta 1865: 133-146, 2016.

22. Khan IS and Ehtesham M: Isolation and characterization of stem cells from human central nervous system malignancies. Adv Exp Med Biol 853: 33-47, 2015.

23. Mattoscio D and Chiocca S: SUMO pathway components as possible cancer biomarkers. Future Oncol 11: 1599-1610, 2015.

24. Amelio I, Landré V, Knight RA, Lisitsa A, Melino G and Antonov AV: Polypharmacology of small molecules targeting the ubiquitin-proteasome and ubiquitin-like systems. Oncotarget 6 : 9646-9656, 2015

25. Yang XJ and Chiang CM: Sumoylation in gene regulation, human disease, and therapeutic action. F1000Prime Rep 5: 45, 2013.

26. Bettermann K, Benesch M, Weis S and Haybaeck J: SUMOylation in carcinogenesis. Cancer Lett 316: 113-125, 2012.

27. Wu Y, Guo Z, Wu H, Wang X, Yang L, Shi X, Du J, Tang B, Li W, Yang L, et al: SUMOylation represses Nanog expression via modulating transcription factors Oct4 and Sox 2. PLoS One 7: e39606, 2012.

28. Wei F, Schöler HR and Atchison ML: Sumoylation of Oct4 enhances its stability, DNA binding, and transactivation. J Biol Chem 282: 21551-21560, 2007.

29. Han X, Wang XL, Li Q, Dong XX, Zhang JS and Yan QC: HIF-1 $\alpha$ SUMOylation affects the stability and transcriptional activity of HIF-1 $\alpha$ in human lens epithelial cells. Graefes Arch Clin Exp Ophthalmol 253: 1279-1290, 2015.

30. Gu J, Fan Y, Liu X, Zhou L, Cheng J, Cai R and Xue S: SENP1 protects against myocardial ischaemia/reperfusion injury via a HIF1 $\alpha$-dependent pathway. Cardiovasc Res 104: 83-92, 2014.

31. Maqbool R, Rashid R, Ismail R, Niaz S, Chowdri NA and Hussain MU: The carboxy-terminal domain of connexin 43 (CT-Cx43) modulates the expression of $\mathrm{p} 53$ by altering miR-125b expression in low-grade human breast cancers. Cell Oncol (Dordr) 38: 443-451, 2015.

32. Fu Y, Shao ZM, He QZ, Jiang BQ, Wu Y and Zhuang ZG Hsa-miR-206 represses the proliferation and invasion of breast cancer cells by targeting Cx43. Eur Rev Med Pharmacol Sci 19: 2091-2104, 2015

33. Mauro V, Carette D, Pontier-Bres R, Dompierre J, Czerucka D, Segretain D, Gilleron J and Pointis G: The anti-mitotic drug griseofulvin induces apoptosis of human germ cell tumor cells through a connexin 43-dependent molecular mechanism. Apoptosis 18: 480-491, 2013. 